\title{
Adaptive Global Exponential Synchronization of Moving Agent Networks with Nonlinear Coupling
}

\author{
Lifu Wang \\ College of Control and Engineering, \\ Northeastern University At \\ Qinhuangdao, \\ Qinhuangdao, P.R. China, 066004 \\ E-mail: wlfkz@qq.com
}

\author{
Xue Peng \\ College of Electrical Information \\ Engineering, Henan Institute of \\ Engineering, \\ Zhengzhou, P.R. China, 451191 \\ E-mail: 85109258@qq.com
}

\author{
Ni Yang \\ Shanghai Institute of Measurement \\ and Testing Technology, \\ Shanghai, P.R. China \\ E-mail: suyejj@163.com
}

\begin{abstract}
- this paper studies the problem of synchronizing a moving agent network by decentralized adaptive control strategy. And, each agent node is assigned nonlinear connections with those of neighboring agents. The moving agent network model exhibits a time-varying topological structure in two dimensional spaces. Base on the Lyapunov stability theory, some criteria for the synchronization are achieved via adaptive control under the constraint of fast switching. To validate the proposed methods, the Lorenz chaotic system as the nodes of the networks are analyzed, and numerical simulations results show the effectiveness of proposed synchronization approaches.
\end{abstract}

Keywords- complex network; exponential synchronization; moving agent; adaptive; controller; nonlinear coupling

\section{INTRODUCTION}

Over the past decade years, the analysis of complex systems from the viewpoint of networks has become an important interdisciplinary issue [1]. Various complex systems such as the Internet, the World Wide Web, electrical power grids, food webs and social systems, can be described as complex networks, where the individual units are abstracted by nodes, and interactions between individuals are represented as edge. These interactions between nodes determine many basic properties of a network. To well understand the complex dynamical behaviors of many natural systems, we need to study their operating mechanism, dynamic behavior, synchronous capabilities, anti-jamming ability, and so on. Among all of the dynamical behaviors of complex networks, synchronization is one of the most interesting topics and has been extensively investigated.

Many studies have pointed out that topological structure plays a significant role in the formation of network synchronization [2, 3]. So far, the great majority of research activities have been focused on static networks, whose topology and coupling configuration in the network are time invariant [4-9]. However, these static connection topologies do not fit most realistic network systems; e.g., in biological, communication, social, and epidemiological networks the connection topology of the network generally changes in time. For these time-varying networks, it is not easy to study the synchronization. Therefore, researchers have recently devoted more and more their efforts to synchronization of time-varying complex networks. A time-varying complex dynamical network model is present by [10], and its synchronization phenomenon is investigated. Stilwell et al. prove that if the network of oscillators synchronizes for the static time-average of the topology, then the network will synchronize with the time-varying topology if the timeaverage is achieved sufficiently fast [11]. How one designs controllers to make dynamical time-varying networks synchronize is a significant issue.

Inspired by the above discussions, in this study the adaptive control problem for a specific time-varying network model is investigated. The model arises from the interaction of mobile agents proposed by [12], and can be widely used to explore various practical problems. In the constraint of fast switching, exponential synchronization criterion can be achieved. By using Lyapunov stability theory, adaptive controllers are designed for global exponential synchronization of moving agent network with time-varying topological structures. The adaptive controllers can ensure that the states of moving agent network fast synchronization.

\section{A Moving Agent Network Model AND PRELIMINARIES}

Consider a complex dynamical network consisting of $N$ identical nodes with nonlinear coupling, in which each node is an n-dimensional dynamical system. The state equations of the network are of the form

$$
\dot{x}_{i}(t)=f\left(x_{i}(t), t\right)-\sigma \sum_{j=1}^{N} g_{i j}(t) h\left(x_{j}(t), t\right)+u_{i}(t)
$$

where $i=1, \cdots, N, x_{i}(t)=\left(x_{i 1}(t), x_{i 2}(t), \cdots, x_{i n}(t)\right)^{T} \in R^{n}$ are the state variables of the node $i ; f: D \times R^{+} \rightarrow R^{n}$ is a smooth nonlinear vector-valued function governs the local dynamics of oscillator; $h: D \times R^{+} \rightarrow R^{n}$ is called the innerlinking function; $\sigma$ is the coupling strength; $u_{i}(t) \in R^{n}$ are the control inputs; $G(t)=\left[g_{i j}(t)\right] \in R^{N \times N}$ is called the outer-coupling matrix or topological matix. This study only considers the case that the network is diffusively connected, i.e., the entries of $G(t)$ satisfy

$$
g_{i i}(t)=-\sum_{j=1, j \neq i}^{N} g_{i j}(t), i=1,2, \cdots, N
$$

Futher, at given time $t$, suppose that if there is an edge 
between node $i$ and node $j$, then $g_{i j}(t)=g_{j i}(t)=-1$, else $g_{i j}(t)=$ $g_{j i}(t)=0$.

It should be noted that, in this study, the each node of network (1) is a moving agent, proposed by [12] distributed in a two-dimensional space of size $L$ with periodic boundary conditions. Each agent moves with velocity $v_{i}(t)$, and direction of motion $\theta_{i}(t)$. The velocity $v_{i}(t)$ is the same for all individuals, (denoted by $v$ ) and is updated in direction through the angle $\theta_{i}(t)$ for each time unit. The agents are considered as random walkers whose positions and orientations are updated according to

$$
\begin{aligned}
& y_{i}\left(t+\Delta t_{M}\right)=y_{i}(t)+v_{i}(t) \Delta t_{M} ; \\
& \theta_{i}\left(t+\Delta t_{M}\right)=\eta_{i}\left(t+\Delta t_{M}\right),
\end{aligned}
$$

Where, $i=1,2, \cdots, N, y_{i}(t)$ is the position of agent $i$ in the plane at time $t, \eta_{i}(t)$ are $N$ independent random variables chosen at each time unit with uniform probability in the interval $[0,2 \pi]$, and $\Delta t_{M}$ is the time unit. Each agent interacts at a given time with only those agents located within a neighborhood of an interaction radius, defined as $R$ $[12,13]$. When two agents interact, there is an edge between them.

In this study, the control objective is to make the states of network (1) globally exponentially synchronize to a manifold defined in (3) by introducing a simple adaptive controller into each individual node.

$$
x_{1}(t)=x_{2}(t)=\cdots=x_{N}(t)=s(t)
$$

where $s(t)$ is a solution of an isolated node,

$$
\dot{s}(t)=f(s(t), t)
$$

We assume that $s(t)$ is an arbitrary desired state which can be an equilibrium point, a periodic orbit, an aperiodic orbit, or even a chaotic orbit in the phase space.

\section{AdAPTIVE SYNCHRONIZATION OF MOVING AGENT NETWORKS}

In this section, we discuss the synchronization of moving agent network (1) by designing adaptive controllers for each agent node. Several network synchronization criteria are given.

In order to achieve the objective of global exponential synchronizaiton on the manifold (3), let us define the error vector

$$
e_{i}(t)=x_{i}(t)-s(t), i=1,2, \cdots N
$$

When the complex network (1) achieves synchronization, the coupling functions and control inputs should vanish, namely, $\sum_{j=1}^{N} g_{i j}(t) h(s(t), t)+u_{i}(t)=0$. This ensures that any solution $f\left(x_{i}(t), t\right)$ of a single isolated node is also a solution of the synchronized coupled network. Subtracting (4) from (1) yields the error dynamical system

$$
\dot{e}_{i}(t)=\bar{f}\left(e_{i}(t), s(t), t\right)-\sigma \sum_{j=1}^{N} g_{i j}(t) \bar{h}\left(e_{j}(t), s(t), t\right)+u_{i}(t)
$$

Where,

$$
\bar{f}\left(e_{i}(t), s(t), t\right)=f\left(x_{i}(t), t\right)-f(s(t), t)
$$

$\bar{h}\left(e_{i}(t), s(t), t\right)=h\left(x_{i}(t), t\right)-h(s(t), t)$.

Then, global exponential synchronization problem of the dynamical network (1) is equivalent to the problem of global exponential stabilization of the error dynamical system (7). In the following, we give several useful hypotheses.

Assumption 1 (A1). Suppose there exists a nonnegative constant $\mu$, satisfying

$$
\left\|\bar{f}\left(e_{i}(t), s(t), t\right)\right\| \leq \mu\left\|e_{i}(t)\right\| .
$$

Assumption 2 (A2). Suppose there exists a nonnegative constant $\mu$, satisfying

$$
\left\|h\left(x_{i}(t), t\right)-h(s(t), t)\right\| \leq \rho\left\|e_{i}(t)\right\|
$$

Assumption 3 (A3). Suppose there exists a constant $T$ such that coupling matrix $G(t)$ satisfies

$$
\frac{1}{T} \int_{t}^{t+T} G(\tau) d \tau=\bar{G}
$$

where, $\bar{G}$ is the time-average of the coupling matrix $G(t)$.

This Assumption 3 implies that the switching between all the possible network configurations is sufficiently fast as defined in [11]. According to [11] the following Lemma can be given.

Lemma 1. Suppose a set of coupled oscillators network with fixed topology defined by equation (8)

$$
\dot{x}_{i}(t)=f\left(x_{i}(t), t\right)-\sigma \sum_{j=1}^{N} \bar{g}_{i j} h\left(x_{j}(t), t\right)+u_{i}(t)\left(\bar{G}=\left[\bar{g}_{i j}\right]\right)
$$

admits a stable synchronization manifold and if (A3) hold. Then the set of coupled oscillators with a time-variant network defined by (1) admits a stable synchronization manifold.

The proof of Lemma 1 can be obtained by main results of [11]. According to analysis of [12], under the constraint of fast switching, $\bar{G}=p G_{A}$, where $p$ is the probability that a link is activated and thus $p=\pi R^{2} / L^{2}$, and $G_{A}$ is the all-toall coupling matrix with zero-row sum). That is

$$
\bar{G}=\frac{\pi R^{2}}{L^{2}}\left[\begin{array}{cccc}
N-1 & -1 & \cdots & -1 \\
-1 & N-1 & \cdots & -1 \\
\vdots & \vdots & \ddots & \vdots \\
-1 & -1 & \cdots & N-1
\end{array}\right]
$$

Based on (A1) and (A2), a network synchronization criterion is deduced as follows.

Theorem 1. Suppose that (A1), (A2) and (A3) hold. Then the dynamical moving agent network (1) is globally exponentially synchronized under the following sets of adaptive controllers

$$
\begin{gathered}
u_{i}=-d_{i} e_{i}, i=1,2, \cdots, N \\
\dot{d}_{i}=e^{2 \lambda t}\left\|e_{i}\right\|^{2},
\end{gathered}
$$

where $d_{i}\left(t_{0}\right)=\mu+\frac{\sigma \pi R^{2}}{L^{2}}\left(N-1+N \frac{\rho^{2}}{2}\right)+\lambda, \mu$ and $\rho$ are defined in the (A1) and (A2), $\lambda$ is the exponential rate available to be designed.

Proof: Select a Lyapunov function as follows 


$$
V(t)=\frac{1}{2} \sum_{i=1}^{N} e_{i}^{T} e_{i}+\frac{1}{2} \sum_{i=1}^{N} e^{-2 \lambda}\left(d_{i}-d_{i}^{*}\right)^{2}
$$

where constant $d_{i}^{*}$ to be given below. Then the time derivative of $V(t)$ along the solution of the error system (7) is given as follows

$$
\begin{aligned}
& \dot{V}=\frac{1}{2} \sum_{i=1}^{N}\left(\dot{e}_{i}^{T} e_{i}+e_{i}^{T} \dot{e}_{i}\right)+\frac{1}{2} \sum_{i=1}^{N} e^{-2 \lambda t}(-2 \lambda)\left(d_{i}-d_{i}^{*}\right)^{2} \\
& +\sum_{i=1}^{N} e^{-2 \lambda t}\left(d_{i}-d_{i}^{*}\right) \dot{d}_{i} \\
& =\frac{1}{2} \sum_{i=1}^{N}\left(\left(\bar{f}\left(e_{i}(t), s(t), t\right)-\sigma \sum_{j=1}^{N} g_{i j}(t) \bar{h}\left(e_{j}(t), s(t), t\right)+u_{i}\right)^{T} e_{i}\right. \\
& \left.+e_{i}^{T}\left(\bar{f}\left(e_{i}(t), s(t), t\right)-\sigma \sum_{j=1}^{N} g_{i j}(t) \bar{h}\left(e_{j}(t), s(t), t\right)+u_{i}\right)\right) \\
& -\lambda \sum_{i=1}^{N} e^{-2 \lambda t}\left(d_{i}-d_{i}^{*}\right)^{2}+\sum_{i=1}^{N} e^{-2 \lambda t}\left(d_{i}-d_{i}^{*}\right) e^{2 \lambda t} e_{i}^{T} e_{i} \\
& =\sum_{i=1}^{N} e_{i}^{T} \bar{f}\left(e_{i}(t), s(t), t\right)-\sigma \sum_{i=1}^{N} \sum_{j=1}^{N} g_{i j}(t) e_{i}^{T} \bar{h}\left(e_{j}(t), s(t), t\right) \\
& -\sum_{i=1}^{N} d_{i}^{*} e_{i}^{T} e_{i}-\lambda \sum_{i=1}^{N} e^{-2 \lambda t}\left(d_{i}-d_{i}^{*}\right)^{2} \\
& \sum_{i=1}^{N} e_{i}^{T} \bar{f}\left(e_{i}(t), s(t), t\right) \leq \mu \sum_{i=1}^{N}\left\|e_{i}^{T}\right\|\left\|e_{i}\right\|=\mu \sum_{i=1}^{N} e_{i}^{T} e_{i}
\end{aligned}
$$

Therefore,

$$
\begin{aligned}
\dot{V} & \leq \mu \sum_{i=1}^{N} e_{i}^{T} e_{i}-\sigma \sum_{i=1}^{N} \sum_{j=1}^{N} g_{i j}(t) e_{i}^{T} \bar{h}-\sum_{i=1}^{N} d_{i}^{*} e_{i}^{T} e_{i} \\
& -\lambda \sum_{i=1}^{N} e^{-2 \lambda t}\left(d_{i}-d_{i}^{*}\right)^{2}
\end{aligned}
$$

Here, if (A3) hold, according to Lemma 1, under the constraint of fast switching, one substitute $g_{i j}(t)$ for $\bar{g}_{i j}$. Then,

$$
\begin{aligned}
\dot{V} \leq & \mu \sum_{i=1}^{N} e_{i}^{T} e_{i}-\sigma \sum_{i=1}^{N} \sum_{j=1}^{N} \bar{g}_{i j} e_{i}^{T} \bar{h}\left(e_{j}(t), s(t), t\right) \\
& -\sum_{i=1}^{N} d_{i}^{*} e_{i}^{T} e_{i}-\lambda \sum_{i=1}^{N} e^{-2 \lambda t}\left(d_{i}-d_{i}^{*}\right)^{2}
\end{aligned}
$$

Therefore,

$$
\begin{aligned}
\dot{V} \leq & \mu \sum_{i=1}^{N} e_{i}^{T} e_{i}+\frac{\sigma \pi R^{2}}{L^{2}}\left(N-1+N \frac{\rho^{2}}{2}\right) \sum_{i=1}^{N} e_{i}^{T} e_{i} \\
& -\sum_{i=1}^{N} d_{i}^{*} e_{i}^{T} e_{i}-\lambda \sum_{i=1}^{N} e^{-2 \lambda t}\left(d_{i}-d_{i}^{*}\right)^{2}
\end{aligned}
$$

Let $d_{i}^{*}=\mu+\frac{\sigma \pi R^{2}}{L^{2}}\left(N-1+N \frac{\rho^{2}}{2}\right)+\lambda$. Therefore,

$$
\dot{V} \leq-\lambda \sum_{i=1}^{N} e_{i}^{T} e_{i}-\lambda \sum_{i=1}^{N} e^{-2 \lambda t}\left(d_{i}-d_{i}^{*}\right)^{2}=-2 \lambda V(t)
$$

By calculating integration on both sides of above inequality, we get $V(t) \leq e^{-2 \lambda\left(t-t_{0}\right)} V\left(t_{0}\right)$.
According (11), one can get $\frac{1}{2} \sum_{i=1}^{N} e_{i}^{T} e_{i} \leq V(t)$, so,

$$
\frac{1}{2} \sum_{i=1}^{N} e_{i}^{T} e_{i} \leq e^{-2 \lambda\left(t-t_{0}\right)} V\left(t_{0}\right) .
$$

Let $d_{i}\left(t_{0}\right)=d_{i}^{*}$, so, $\frac{1}{2} \sum_{i=1}^{N} e_{i}^{T} e_{i} \leq e^{-2 \lambda\left(t-t_{0}\right)} \frac{1}{2} \sum_{i=1}^{N} e_{i}^{T}\left(t_{0}\right) e_{i}\left(t_{0}\right)$.

That means, $\|e(t)\|^{2} \leq e^{-2 \lambda\left(t-t_{0}\right)}\left\|e\left(t_{0}\right)\right\|^{2}$.

So, $\|e(t)\| \leq \alpha e^{-\lambda t}$, where $\alpha=e^{\lambda t_{0}}\left\|e\left(t_{0}\right)\right\|$.

Therefore, in closed-loop under the controllers (10), it follows that the error system (7) is globally exponentially stable at the equilibrium set $e_{i}=0, i=1,2, \cdots N$, with the exponential rate $\lambda$. Consequently, the synchronous solution of the dynamical network (1) is globally exponentially stable. The proof is thus completed.

\section{Simulation}

In this section, one example is given for illustrating the proposed synchronization criteria. Consider a dynamical network consisting of 5 identical Lorenz oscillators. Where, state dynamics of each agent is described by

$$
\left(\begin{array}{l}
\dot{x}_{i 1} \\
\dot{x}_{i 2} \\
\dot{x}_{i 3}
\end{array}\right)=\left(\begin{array}{ccc}
-10 & 10 & 0 \\
28 & -1 & 0 \\
0 & 0 & -8 / 3
\end{array}\right)\left(\begin{array}{c}
x_{i 1} \\
x_{i 2} \\
x_{i 3}
\end{array}\right)+\left(\begin{array}{c}
0 \\
x_{i 1} x_{i 3} \\
x_{i 1} x_{i 2}
\end{array}\right),
$$

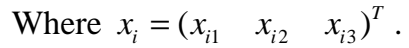

Each agent node interacts at a given time with only those agents located within a neighborhood of an interaction radius. Here, we let interaction radius $R=40$, and periodic boundary conditions size $L=80$. The initial position $y_{i}(0)$ of agent $i$ in the plane is choose at random, The initial orientation $\theta_{i}(0)=0$, other time unit chosen at each time unit with uniform probability in the interval, The each agent mobile velocity $v=100$, each time unit $\Delta t_{M}=0.1$, each agent moving 50 time unit $(\mathrm{T}=50)$.

When two agents interact, the state equations of each agent are changed to include diffusive coupling with the neighboring agent, acting on the following equations:

$$
\dot{x}_{i}=f\left(x_{i}\right)-\sigma \sum_{j=1}^{N} g_{i j}(t) h\left(x_{j}\right)+u_{i}
$$

where, $i=1, \cdots, N, f: R^{3} \rightarrow R^{3}$ is given by the Lorenz dynamics, $h\left(x_{i}\right)=\left[\begin{array}{lll}0 & x_{i 2} & x_{i 1} x_{i 3}\end{array}\right]^{T}, g_{i j}(t)$ are the elements of a time-varying matrix $G(t)$, and $u_{i}=-d_{i} e_{i}$, $\dot{d}_{i}=e^{2 \lambda t}\left\|e_{i}\right\|^{2}$.

Similar to [14, 15], since Lorenz chaotic system has a chaotic attractor which is confined to abounded region $\phi \subset R^{n}$ [16], there exists a constant $M$ satisfying $\left|x_{i j}\right|,\left|s_{j}\right| \leq M$ for $i=1,2, \cdots, N$ and $j=1,2,3$. Therefore

$$
\left\|\bar{h}\left(x_{i}, s, t\right)\right\|=\sqrt{\left(x_{i 2}-s_{2}\right)^{2}+\left(x_{i 1} x_{i 3}-s_{1} s_{3}\right)^{2}} \leq 2 M\left\|e_{i}\right\| .
$$

$M$ can be got from the method of similar to [16]. Thus, (A3) 
hold.

According to Theorem 2, the synchronous solution $s(t)$ of dynamical moving agent network (15) is globally exponentially stable. The other parameters are assigned as follows: $k_{i}=1, d_{i}(0)=1, x_{i}(0)=(2+0.5 i, 3+0.5 i, 4+0.5 i)$, and $\sigma=1$. The synchronous error $e_{i}$ are shown in Figure 24. Obviously, the zero error is globally exponentially stable for dynamical network (15).

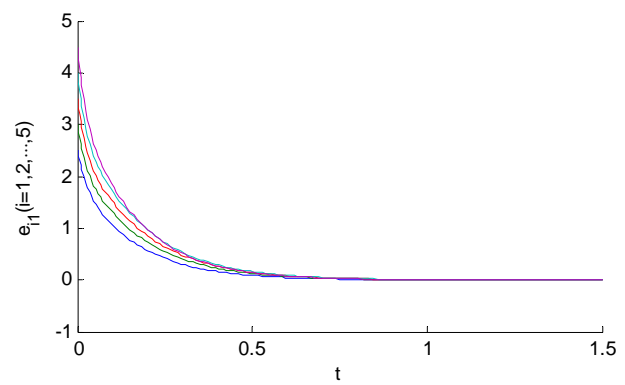

Figure 2. Synchronization errors of $e_{i 1}$ for the network

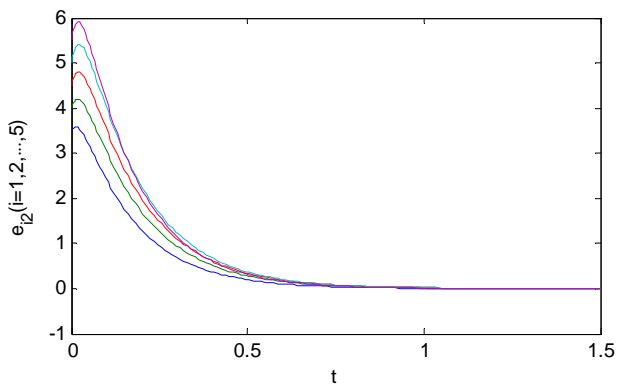

Figure 3. Synchronization errors of $e_{i 2}$ for the network.

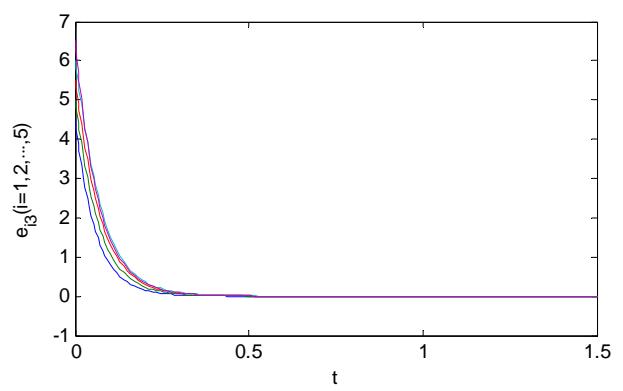

Figure 4. Synchronization errors of $e_{i 3}$ for the network.

\section{CONCLUSION}

The problem of global exponential synchronization for moving agent dynamical network is investigated. The complex network with nonlinear coupling is considered as a large-scale nonlinear system. A Lyapunov function is constructed to deal with the problem of controlled synchronization as to ensure the closed loop system stability. Then, a novel network synchronization criterion has been proved. Decentralized adaptive controllers are designed to achieve synchronization for the moving agent networks.
Compared with some similar results, the proposed adaptive controllers are simple and effective to implement. It can achieve quickly asymptotically synchronization. And a numerical simulation of coupled Lorenz system network is given, which demonstrates the effectiveness of the proposed methods.

\section{ACKNOWLEDGMENT}

This work is supported by by the Natural Science Foundation of Hebei under Grant No. F2012501030, the Fundamental Research Funds for the Central Universities under Grant \#N100323012.

\section{REFERENCES}

[1] R. Albert and A. L. Barabasi, "Statistical mechanics of complex networks," Reviews of Modern Physics, vol. 74, pp. 47-97, Jan 2002.

[2] A. Arenas, et al., "Synchronization in complex networks," Physics Reports, vol. 469, pp. 93-153, 2008.

[3] L. M. Pecora and T. L. Carroll, "Master stability functions for synchronized coupled systems," Physical Review Letters, vol. 80, pp. 2109-2112, Mar 91998.

[4] M. Barahona and L. M. Pecora, "Synchronization in Small-World Systems," Physical Review Letters, vol. 89, p. 054101, 2002.

[5] X. F. Wang and G. R. Chen, "Synchronization in scale-free dynamical networks: Robustness and fragility," Ieee Transactions on Circuits and Systems I-Fundamental Theory and Applications, vol. 49, pp. 54-62, Jan 2002.

[6] S. Boccaletti, et al., "Complex networks: Structure and dynamics," Physics Reports-Review Section of Physics Letters, vol. 424, pp. 175308, Feb 2006

[7] W. Guo, "Lag synchronization of complex networks via pinning control," Nonlinear Analysis: Real World Applications, vol. 12, pp. 2579-2585, 2011.

[8] J. G. Barajas-Ramírez, "Robust synchronization of a class of uncertain complex networks via discontinuous control," Computers and Mathematics with Applications, p. doi:10.1016/j.camwa.2012.01.082, 2012.

[9] S. Zheng, et al., "Adaptive synchronization of two nonlinearly coupled complex dynamical networks with delayed coupling," Communications in Nonlinear Science and Numerical Simulation, vol. 17, pp. 284-291, 2012.

[10] J. H. Lu and G. R. Chen, "A time-varying complex dynamical network model and its controlled synchronization criteria," Ieee Transactions on Automatic Control, vol. 50, pp. 841-846, Jun 2005.

[11] D. J. Stilwell, et al., "Sufficient conditions for fast switching synchronization in time-varying network topologies," Siam Journal on Applied Dynamical Systems, vol. 5, pp. 140-156, 2006.

[12] M. Frasca, et al., "Synchronization of Moving Chaotic Agents," Physical Review Letters, vol. 100, p. 044102, 2008.

[13] L. Wang, et al., "Induced synchronization of a mobile agent network by phase locking," Physical Review E, vol. 82, p. 046222, 2010.

[14] K. Li and C. Lai, "Adaptive-impulsive synchronization of uncertain complex dynamical networks," Physics Letters A, vol. 372, pp. 16011606, 2008.

[15] J. Zhou, et al., "Adaptive synchronization of an uncertain complex dynamical network," Ieee Transactions on Automatic Control, vol. 51 , pp. 652-656, Apr 2006.

[16] D. Li, et al., "Estimating the ultimate bound and positively invariant set for the Lorenz system and a unified chaotic system," Journal of Mathematical Analysis and Applications, vol. 323, pp. 844-853, 2006. 